\title{
Pediatría
}

http://www.revistapediatria.org/

Regional Bogotá

DOI: https://10.14295/rp.v53i4.210

\section{Quiste aracnoideo gigante ¿Debe el pediatra alarmarse? a propósito de un caso asociado a hemorragia intraquística espontánea.}

\section{Mónica-Paola Gutiérrez-Gaitán ${ }^{a}$, Andrés-David Montoya-Moncada ${ }^{b}$, Javier-Yesid Pinzon- Salamancac,f, Mónica Cediel-Echeverrid,f, José-Miguel Suescun-Vargas ${ }^{e, f}$}

a. Médico, Universidad del Bosque. Bogotá Colombia.

b. Médico, Universidad del Rosario. Bogotá Colombia.

c. Coordinador, Programa de Hospitalización Domiciliaria Instituto Roosevelt, Bogotá, Colombia.

d. Pediatra, Universidad Militar. Bogotá Colombia.

e. Pediatra de Hospitalización, Instituto Roosevelt. Bogotá Colombia.

f. Profesores de pediatría Universidad del Rosario y Universidad de los Andes. Bogotá, Colombia.

INFORMACIÓN DEL ARTÍCULO

Historia del artículo:

Recibido el 29 de octubre 2019

Aceptado el 12 de marzo 2021

\section{Palabras clave:}

Quistes Aracnoideos.

hemorragias intracraneales.

cefalea.
R E S U M E N
Keywords:

Arachnoid Cysts.

intracranial hemorrhages.

headache.

\begin{abstract}
Antecedentes: Los quistes aracnoideos son masas benignas, uniloculares, compuestas por membrana colágena, revestida por células aracnoideas, con liquido céfalo-raquídeo en su interior. Reporte de caso: se presenta el caso clínico; niño de 9 años con síntomas de cefalea, náuseas y vómito, con posterior diagnóstico de quiste aracnoideo gigante parieto-temporofrontal izquierdo, con desplazamiento de línea media hacia la derecha con hemorragia intraquística espontanea documentada por neuroimagen (resonancia magnética cerebral sin contraste), sin presentar cambios comportamentales, convulsiones, o déficit del estado neurológico. Se realiza abordaje quirúrgico con craneotomía y drenaje de colección hemática subdural que comprimía parénquima adyacente con sospecha de Fistula de líquido céfaloraquídeo sin signos de hipertensión endocraneana. Conclusiones: Actualmente se ha incrementado la incidencia de quistes aracnoideos en pacientes pediátricos, siendo evidentes desde el tercer trimestre de gestación, con predominio de frecuencia en hombres y del hemisferio izquierdo. Se realiza un enfoque clínico pediátrico para un adecuado abordaje.
\end{abstract}

Giant arachnoid cyst ¿Should the pediatrician be alarm? Case report associated with spontaneous intracystic hemorrhage.

A B S T R A C T

Background: Arachnoid cysts are benign unilocular masses composed of a collagenous membrane, lined by arachnoid cells, with cerebrospinal fluid. Case report: We present the case of a 9-year-old boy with headache, nausea, and vomiting, with a subsequent diagnosis of a giant arachnoid cyst parietal-temporal-left frontal, with a deviation of the midline to the right, with spontaneous intracystic hemorrhage documented by neuroimaging (brain magnetic resonance

\footnotetext{
*Autor para correspondencia. Mónica Paola Gutiérrez Correo electrónico: moni18gutierrez@gmail.com
}

Como Citar: Gutiérrez MP, Montoya A, Pinzon-Salamanca JY, Cediel Echeverri M, Suescun-Vargas JM. Quiste aracnoideo gigante ¿Debe el pediatra alarmarse? a propósito de un caso asociado a hemorragia intraquística espontánea. Pediatr. 2020;53(4):158-161. 
imaging without contrast), without behavioral changes, seizures, or deficits in neurological status. A surgical approach was performed with craniotomy and drainage of a subdural blood collection that compressed the adjacent parenchyma with suspected cerebrospinal fluid fistula without signs of intracranial hypertension. Conclusions: Currently, the incidence of arachnoid cysts has increased in pediatric patients, being evident from the third trimester of gestation, with a high prevalence in men and the left hemisphere. A pediatric clinical approach is performed

\section{Introducción}

Dentro de las lesiones quísticas intracraneales, no neoplásicas, se encuentran los quistes aracnoideos (QA), que son masas de carácter benigno, y en su mayoría, uniloculares, compuestas microscópicamente de una membrana colágena, la cual, se encuentra revestida por células aracnoideas, con líquido cefalorraquídeo (LCR) en su interior (1). Los QA, tradicionalmente se han entendido como lesiones congénitas, originadas de una disgenesia en el desarrollo embriológico que conduce a la división o duplicación de la membrana aracnoidea $(1,2,3)$. Sin embargo, existe la posibilidad de que su formación de novo, sea debida a una acumulación posinflamatoria de LCR en el espacio subaracnoideo posterior a un trauma, infección intracraneal o hemorragia, dando lugar a los QA secundarios $(1,2,4)$. En la población pediátrica la prevalencia es de $2.6 \%$ (5), correspondiendo al $1 \%$ de todas las lesiones intracraneales (3).

Su localización más frecuente es la fosa craneal media (5). Dos terceras partes ocupan el espacio supratentorial y la mitad se encuentra en relación con la cisterna de Silvio (3). Otros lugares incluyen la cisterna supraselar, la fosa posterior (cisterna pontocerebelosa), dentro de la fisura interhemisférica, sobre la convexidad cerebral, en la fisura coroidea, cisterna magna, cisterna cuadrigeminal o fisuras vermianas (1). Galassi y cols. (6) han descrito una clasificación radiológica, ampliamente aceptada de los QA de fosa media, Tipo I: Quistes ubicados en la cara anterior del lóbulo temporal sin efecto de masa. Tipo II: Quistes de tamaño medio situados en la parte anterior y media de la fosa temporal, que comprimen el lóbulo temporal y Tipo III: Quistes de gran tamaño, de forma oval o redonda, que abarcan la totalidad de la fosa temporal, con efecto de masa.

Dentro de la historia natural de la enfermedad, son pocos los casos que presentan manifestaciones clínicas, y que a su vez, comprenden un gran espectro clínico según su localización y tamaño (1,3), desde síntomas inespecíficos como cefalea, sensación vertiginosa, vómito, proptosis, epilepsia, trastorno por déficit de atención e hiperactividad (TDAH), déficit cognitivo o de lenguaje, retraso en el desarrollo psicomotor, trastornos endocrinos; u otros más complejos relacionados con efecto de masa como hidrocefalia obstructiva, síndrome de hipertensión endocraneana y focalización neurológica (2).

La gran mayoría de casos son asintomáticos, siendo diagnosticados como hallazgo incidental en ultrasonografía, tomografía axial computarizada (TAC) y resonancia magnética nuclear (RMN) cerebral (1). Por la baja prevalencia de la patología y el pobre reporte de casos en nuestro país, los QA representan un reto clínico para el pediatra tanto en el diagnóstico como en el tratamiento. Con la presentación de este caso con diagnóstico de QA, con hemorragia intraquística asociada, se pretende dar un enfoque con respecto a identificación y abordaje adecuado de esta patología.

\section{Reporte de caso}

Paciente masculino de 9 años, previamente sano, quien consulta por cuadro de dos días de evolución de primer episodio de cefalea de localización frontal de intensidad moderada a severa, tipo pulsátil, asociado a fotofobia, náuseas y vómito. Sin antecedentes de trauma o proceso infeccioso. Sin respuesta al manejo analgésico convencional. Se hospitaliza para toma de TAC de cráneo simple, en el que se describe desviación de línea media hacia la derecha sin claridad de lesión ocupando espacio o signos de hipertensión endocraneana.

Se amplían estudios con RNM cerebral sin contraste, evidenciando gran quiste parieto- temporo-frontal izquierdo, con desplazamiento de la línea media hacia la derecha, con obliteración parcial de ventrículo lateral izquierdo y de cisterna crural izquierda, con efecto expansivo extra axial hemisférico izquierdo de características quísticas con contenido hemático, con hallazgo incidental de sinusitis etmoidal (Ver figura 1).

Valorado por el servicio de neurocirugía, que considera quiste aracnoideo temporal izquierdo grado III en la clasificación de Galassi, con signos de hemorragia subaguda en su interior. Se inicia tratamiento antibiótico con amoxicilina por la sinusitis etmoidal para posterior procedimiento quirúrgico por riesgo de hipertensión endocraneana.

Por craneotomía fronto-temporo-parietal izquierda se evidencia colección hemática subdural. Se confirma presencia de quiste aracnoideo gigante que ocupa la totalidad de la fosa media, de forma parcial la fosa anterior izquierda, fosa posterior y cisternas supraselares, que desplaza hacia la derecha y comprime parénquima adyacente. Después del drenaje es enviado a unidad de cuidados intensivos para monitorización neurológica. Nunca se documentó cambios comportamentales, convulsiones o déficit del estado neurológico.

El TAC cerebral simple de control en el día uno del posoperatorio, sin evidencia de sangrado, sin desviación de la línea media. El control de los 10 días, con TAC cerebral simple con colección hipodensa subgaleal fronto-temporo-parietal izquierda, presentó una imagen residual de quiste aracnoideo temporal grado III sin efecto de masa, sin desviación de línea media.

El paciente persiste sin deterioro neurológico, por sospecha clínica de fistula contenida de LCR, sin signos de hipertensión endocraneana, se realiza punción y drenaje subcutáneo con vendaje cefálico compresivo sin complicaciones. El paciente egresó con vendaje compresivo cefálico, signos de alarma y control por neurocirugía y pediatría. 


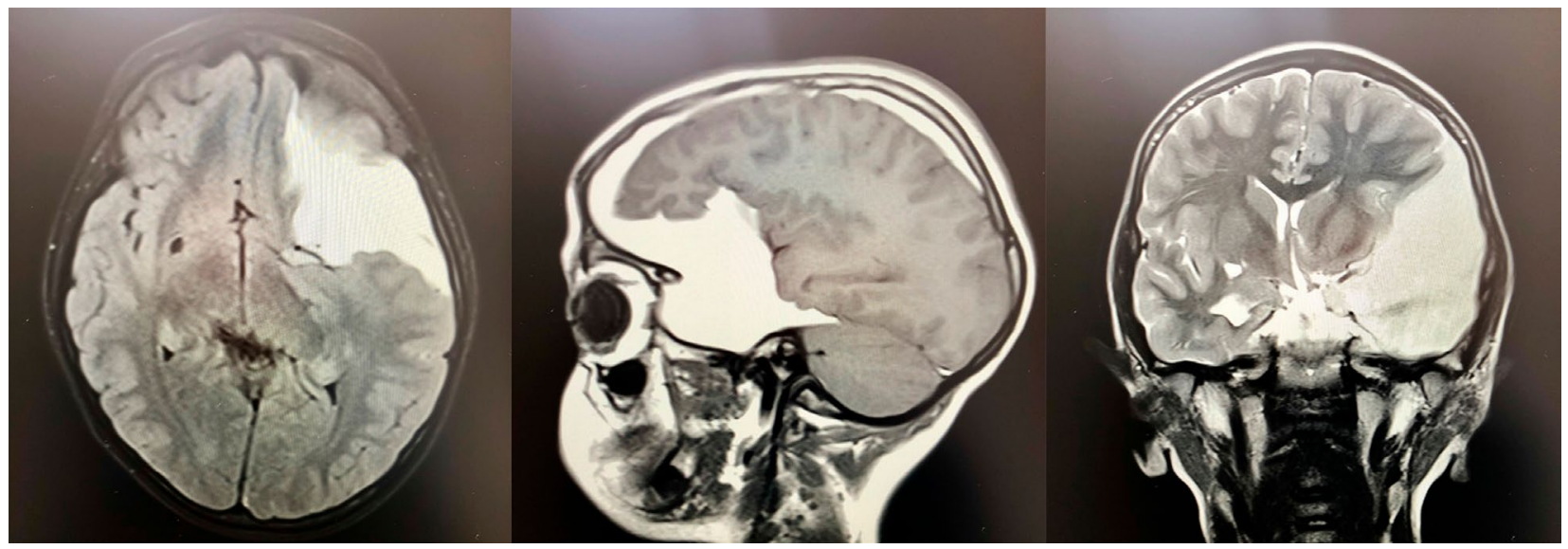

Figura 1. Resonancia Nuclear Magnética Cerebral.

Se evidencia gran quiste parieto- temporo-frontal izquierdo, se aprecia el desplazamiento de la línea media hacia la derecha. Obliteración parcial de ventrículo lateral izquierdo y de cisterna crural izquierda. Efecto expansivo extra axial hemisférico izquierdo de características quísticas con contenido hemático

\section{Discusión}

En los últimos años, ha incrementado la incidencia de los QA, por aumento en acceso a neuroimágenes (7). Desde el mismo tamizaje prenatal la mayoría de los QA, son evidentes en el tercer trimestre, como lesiones anecoicas regulares, ubicadas en el área supratentorial (8). En la TAC, aparecen masas con hipodensidad uniforme coincidente con el LCR; así como en la RMN, con intensidad de señal de LCR en todas las secuencias de pulso, hipointensa en T1W, hiperintensa T2W y supresión completa en secuencia FLAIR (9), concordante con las imágenes de nuestro caso.

En la TAC, el hueso adyacente puede ser delgado por erosión por presión. Un cambio en la neuroimagen dentro o alrededor del QA sugiere una hemorragia reciente, como la del caso (9). Los QA son más frecuentemente diagnosticados en la infancia, con preponderancia en hombres (2) y compromiso del hemisferio izquierdo (4). En pacientes sintomáticos, la cefalea es el síntoma más común (4). Los pacientes que no han presentado cierre de suturas evidencian aumento del perímetro cefálico, macrocefalia y abultamiento asimétrico del cráneo y en pacientes mayores manifestaciones secundarias a la compresión o irritación por efecto de masa (3). La clínica se correlaciona con la localización, los QA supraselares, asociados a desarrollo de hidrocefalia obstructiva por compresión del foramen de Monroe, manifestaciones visuales y trastornos endocrinos (10), que tienden a persistir, incluso aumentar después de cirugía (11). El TDAH, los desórdenes cognitivos y de desarrollo psicomotor están asociados a QA de fosa media, por hipoperfusión sostenida secundaria al efecto de masa (12).

La severidad de los síntomas depende del aumento de la presión intracraneal, no del tamaño del quiste (13). Se ha reportado que entre el 2.6 al $4.6 \%$ de los QA pueden presentar complicaciones secundarias a la ruptura de su pared (14), con aparición de higromas, con menor frecuencia de hematomas subdurales ocasionados por sangrado de vasos leptomeníngeos (9) ocasionando efecto compresivo. Las complicaciones se rela- cionan con trauma craneoencefálico (TCE), reportando casos de ruptura espontánea. Los factores de riesgo son TCE mayor y tamaño del quiste con diámetro superior a 5 centímetros (14). La mayoría de QA son estables en el tiempo. Niños seguidos más de 30 meses, muestran alta tasa de estabilidad con poca probabilidad de aumento de tamaño (7).

Es inusual el aumentado tamaño relacionado con empeoramiento clínico o radiológico, especialmente en niños mayores (5). Quistes de mayor tamaño tienen alta probabilidad de aumento de volumen a una edad más temprana (15), especialmente en menores de 1 año (7), con relación inversamente proporcional entre la edad y cambios clínico-radiológicos en QA.

En el escenario de aumento de tamaño del quiste, asintomático, no requiere tratamiento quirúrgico y debe hacerse seguimiento estricto (7). El crecimiento aislado sin clínica no es signo de alarma. Hay casos descritos de disminución del tamaño y resolución espontánea como resultado de ruptura de la membrana quística al generar dispersión y reabsorción del LCR en el espacio subdural o subaracnoideo; secundario a trauma o infección intracraneal (15). La mayoría de QA, requieren seguimiento imagenológico, los asintomáticos no requieren tratamiento quirúrgico (1). La cirugía está indicada en: hipertensión endocraneal, hidrocefalia, síntomas focales y hemorragias intraquísticas $(3,12)$. Hay controversia en el resto de los escenarios clínicos, requiriendo análisis por parte del cirujano con escalas validadas de calidad de vida y dolor (13).

\section{Conclusiones}

Los QA en niños son masas de carácter benigno, que pueden generar una clínica muy variable. En general, pueden pasar asintomáticos, sin embargo, algunas veces pueden generar síntomas de alarma y requerir un manejo especializado. Debemos tener muy en cuenta estos signos de alarma ante un paciente con cefalea en la consulta pediátrica, para que esta patología no pase desapercibida. 


\section{Conflictos de interés}

Todos los autores declaran que no existen conflictos de intereses.

\section{Financiación}

Todos los autores declaran que no se recibió ningún tipo de ayuda económica para la realización del manuscrito.

\section{Agradecimientos}

Al Instituto Roosevelt por facilitarnos el acceso a la información.

\section{R E F E R E N C I A S}

1. Taillibert S, Le Rhun E. Chamberlain M Intracranial Cystic Lesions: A Review. Curr Neurol Neurosci Rep. 2014;14(9):481.

2. Hall S, Smedley A, Sparrow O, Mathad N, Waters R, Chakraborty A, Tsitouras V. Natural History of Intracranial Arachnoid Cysts. World Neurosurg. 2019;126:e1315-e1320.

3. Vega-Sosa A, de Obieta-Cruz E, Hernández-Rojas M. Quistes aracnoideos intracraneales, Cir Cir 2010;78:556-562.

4. Gosalakkal J. Intracranial Arachnoid Cysts in Children: A Review of Pathogenesis, Clinical Features, and Management. Pediatr Neurol. 2002;26(2):93-8.

5. Al-Holou W, Yew A, Boomsaad Z, Garton H, Muraszko K, Maher C. Prevalence and natural history of arachnoid cysts in children, J Neurosurg Pediatr. 2010;5(6):578-85.

6. Galassi E, Fiazza G, Gaist G, Frank F. Arachnoid cysts of the middle cranial fossa: a clinical radiological study of 25 cases treated surgically. Surg Neurol. 1980;14(3):211-9
7. Lee J, Kim J, Hoon Phi J, Kim S, Cho B. Wang K. Enlarging arachnoid cyst: a false alarm for infants, Childs Nerv Syst. 2012;28(8):1203-11.

8. Contro E, De Musso F, Pli G, Ghi T. Intracranial Hemorrhagic Cysts Tumors and Destructive Lesion en Obstetric imaging, Copel J. editor, 2nd ed, Elsevier; 2012, P 234-242.

9. Toh C, Castillo M. Intracranial Cysts and Cyst-Like Lesions (Non-Neoplastic, Non-Infectious Cysts) en Imaging of The Brain, Naidich TP, Castillo M, Cha S. Smirniotopoulos J, Editors. Elsevier/Saunders, Philadelphia, 2013, P 629. Chapter 27.

10. Abou-Hamden A, James M, Drake. Hydrocephalus and arachnoid cyst en Swaiman's Pediatric Neurology. Kenneth F, Ashwal S, Ferriero D, Schor N, et al., Editors. Elsevier, 6thed, 2016, chapter 30, P 561-576.

11. Lee J, Lee Y, Jung H, Chong S, Et Al. Long-term endocrine outcome of suprasellar arachnoid cysts. J Neurosurgery Pediatric 2017, 19:696-702.

12. Sgouros S, Chapmanb S. Congenital Middle Fossa Arachnoid Cysts May Cause Global Brain Ischaemia: A Study with 99TcHexamethylpropyleneamineoxime Single Photon Emission Computerised Tomography Scans. Pediatr Neurosurg. 2001;35(4):188-94

13. Mørkve S, Helland C, Amus J, Lund-Johansen M, Western K. Surgical Decompression of Arachnoid Cysts Leads to Improved Quality of Life: A Prospective Study. Neurosurgery. 2016 May;78(5):613-25

14. Cress M, Kestle J, Holubkov R, Riva-Cambrin J. Risk Factors for Pediatric Arachnoid Cyst Rupture/Hemorrhage: A Case-Control Study. Neurosurgery. 2013 May;72(5):716-22

15. Lim J, Choi S, Son S, Kwon H, Koh H, You J. Is arachnoid cyst a static disease? A case report and literature review. Childs Nerv Syst. 2019 Feb;35(2):385-388 